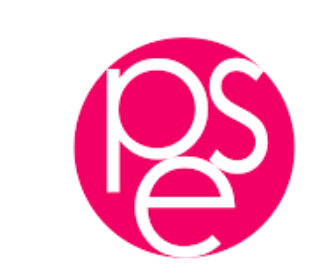

PARISSCHOOLOFECONOMICS
ECOLED'ECONOMIE DE PARIS

WORKING PAPER Nº $2018-43$

\title{
Super-inertial interest rate rules are not solutions of Ramsey optimal monetary policy
}

\author{
Jean-Bernard Chatelain \\ Kirsten Ralf
}

JEL Codes: C61, C62, E43, E44, E47, E52, E58

Keywords : New-Keynesian model, Ramsey optimal policy, Interest rate smoothing, Super-inertial rule, Inertial rule
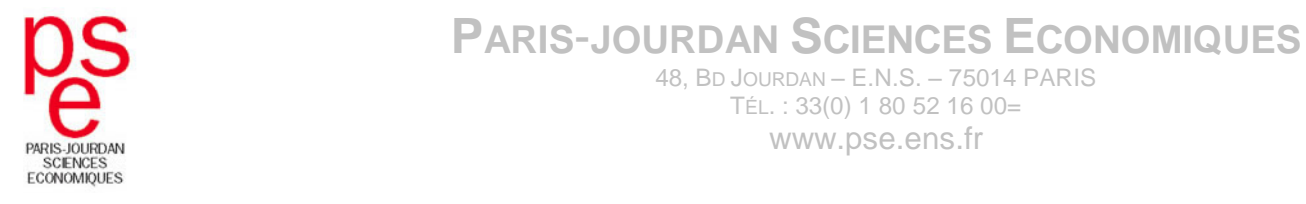


\title{
Super-inertial interest rate rules are not solutions of Ramsey optimal monetary policy
}

\author{
Jean-Bernard Chatelain*and Kirsten Ralf ${ }^{\dagger}$
}

August 27, 2018

\begin{abstract}
Giannoni and Woodford (2003) found that the equilibrium determined by commitment to a super-inertial rule (where the sum of the parameters of lags of interest rate exceed ones and does not depend on the auto-correlation of shocks) corresponds to the unique bounded solution of Ramsey optimal policy for the new-Keynesian model. By contrast, this note demonstrates that commitment to an inertial rule (where the sum of the parameters of lags of interest rate is below one and depends on the auto-correlation of shocks) corresponds to the unique bounded solution.
\end{abstract}

JEL classification numbers: C61, C62, E43, E44, E47, E52, E58.

Keywords: new-Keynesian model, Ramsey optimal policy, interest rate smoothing, super-inertial rule, inertial rule.

\section{Introduction}

Giannoni and Woodford (2003), henceforth GW, found that Ramsey optimal monetary policy under commitment miminizing an infinite horizon discounted loss function including the variance of the interest rates subject to the new-Keynesian model has its unique bounded equilibrium solution determined by commitment from date $t$ onwards to a "super-inertial" interest rate rule where the sum of parameters of the lags of interest rate is strictly above one. This super-inertial policy rule does not depend on the auto-correlation of shocks (Giannoni (2014), p.123).

In recent new-Keynesian dynamic stochastic general equilibrium models of research departments of central banks, GW result provides a rationale for computing super-inertial optimal simple rules without setting a restriction on the sum of parameters for lagged funds rate to be below one when using simulation grids (Adolfson et al. (2014), Chung et al. (2015)). By contrast, positive restrictions and upper limit restrictions are usually set on the parameters for the response of funds rate to inflation and to output gap in simulation grids.

This note demonstrates that the unique bounded equilibrium solution of GW model is determined by commitment from date $t$ onwards to an inertial policy rule, where the

\footnotetext{
*Paris School of Economics, Université Paris I Pantheon Sorbonne, 48 Boulevard Jourdan 75014 Paris. Email: jean-bernard.chatelain@univ-paris1.fr

${ }^{\dagger}$ ESCE International Business School, 10 rue Sextius Michel, 75015 Paris, Email: Kirsten.Ralf@esce.fr.
} 
sum of parameters of the lags of interest rate is strictly below one and depends on the auto-correlation parameters of shocks.

GW super-inertial policy rule is a necessary first order Euler condition but not a sufficient condition to the unique bounded solution. It corresponds no only to the unique bounded solution but also to an infinity of out-of-equilibrium unbounded paths with dynamics for the funds rate which are explosive. For those paths, the discounted variance of funds rate included into the loss function tends to infinity when the growth rate of funds rate exceeds the households discount factor. Those paths maximizes the Central Bank loss function instead of minimizing it. GW super-inertial policy rule may not be a relevant advice to practitioners in Central Banks and in the IMF.

This super-inertial policy rule is one equation of the Hamiltonian system of Ramsey optimal policy. This Hamiltonian system has a saddle-point equilibrium which satisfies Blanchard and Kahn (1980) determinacy condition (GW proposition 1). After proposition 1, GW stop half-way in the computation of optimal policy.

Once the determinacy condition is fulfilled (GW proposition 1), Blanchard and Kahn (1980) and Vaughan (1970) demonstrated that the unique bounded solution and the optimal policy rule are obtained seeking the stable subspace of the Hamiltonian system using the method of undetermined coefficients or, equivalently, solving a matrix Riccati equation which amounts to seek the eigenvectors of stable roots of a Jordan decomposition of the Hamiltonian matrix. This is also demonstrated by Anderson et al. (1996) section 5, by Hansen and Sargent (2008) section 3.5, by Ljungqvist and Sargent (2012) chapter 19. Closer to GW model, this computation is done by Clarida, Gali, Gertler (1999) and by Gali (2015) chapter 5 on a smaller Hamiltonian system included into GW Hamiltonian system.

Section two details the Hamiltonian system of Ramsey optimal policy and GW superinertial rule. Section three presents its solution. Section four concludes.

\section{The Hamiltonian system of Ramsey optimal policy}

GW apply Ramsey optimal policy on the new-Keynesian monetary policy transmission mechanism. The new-Keynesian private sector's four-equations model is written with all variables as log-deviations of an equilibrium. In the representative household's intertemporal substitution consumption Euler equation, current output gap $x_{t}$ is positively correlated with expected output gap and negatively correlated with the real rate of interest, equal to the nominal rate $i_{t}$ minus expected inflation $E_{t} \pi_{t+1}$. The intertemporal elasticity of substitution (IES) $\sigma$ is a measure of the responsiveness of the growth rate of consumption to the interest rate, usually considered to be smaller than one. It is the inverse or the relative degree of resistance to intertemporal substitution of consumption (RISC, the relative fluctuation aversion), which measure the strength of the preference for smoothing consumption over time, usually considered to be larger than one. We keep GW's notation $\sigma$ for the IES, although some other papers denote it $\sigma^{-1}$.

$$
x_{t}=E_{t} x_{t+1}-\sigma\left(i_{t}-E_{t} \pi_{t+1}\right)+z_{x, t} \text { where } \sigma>0 .
$$

An exogenous stationary and predetermined shock $z_{t}$ is auto-regressive of order one $\left(0<\rho_{z}<1\right)$ with given initial value. Disturbances $\varepsilon_{z, t}$ are zero-mean, independently and identically normally distributed. 


$$
z_{t}=\rho_{z} z_{t-1}+\varepsilon_{z, t} \text { where } \varepsilon_{z, t} \text { is i.i.d. } N\left(0, s_{x}^{2}\right), z_{0} \text { given. }
$$

In the new-Keynesian Phillips curve, current inflation is positively correlated with discounted expected inflation and to current output, with a slope parameter $\kappa$.

$$
\pi_{t}=\beta E_{t} \pi_{t+1}+\kappa x_{t}+u_{t} \text { where } \beta>0 \text { and } \kappa>0
$$

An exogenous stationary and predetermined variable cost-push shock $u_{t}$ is autoregressive of order one $\left(0<\rho_{u}<1\right)$ with given initial value. Disturbances $\varepsilon_{u, t}$ are zero-mean, independently and identically normally distributed.

$$
u_{t}=\rho_{u} u_{t-1}+\varepsilon_{u, t} \text { where } \varepsilon_{u, t} \text { is i.i.d. } N\left(0, s_{\pi}^{2}\right), u_{0} \text { given. }
$$

The policy maker minimizes the expectation of the present value of a discounted quadratic loss function by a discount factor $\beta$. The relative weight of inflation variance is set to one. The relative weight on the second policy target (output gap) is positive $\lambda_{x} \geq 0$. The relative weight on the volatility of her policy instrument (interest rate variance) is a strictly positive $\lambda_{i}>0$ :

$$
-E_{t} \sum_{t=0}^{+\infty} \beta^{t}\left\{\frac{\pi_{t}^{2}}{2}+\lambda_{x} \frac{x_{t}^{2}}{2}+\lambda_{i} \frac{i_{t}^{2}}{2}\right\}
$$

This loss function is subject to the private sector's new-Keynesian four equations model (equations $(1,2,3,4)$ ). This is a discounted linear quadratic regulator (LQR) augmented by two forcing variables $\left(z_{t}, u_{t}\right)$ (Hansen and Sargent (2008), section 3.5). A quadratic loss function including a discount factor is minimized subject to a linear dynamic system with boundary conditions.

Denoting Lagrangian multipliers $\phi_{x, t}$ for the consumption Euler equation (1) and $\phi_{\pi, t}$ for the new-Keynesian Phillips curve (3), the Lagrangian $\mathcal{L}$ is:

$\mathcal{L}=-E_{0} \sum_{t=0}^{+\infty} \beta^{t}\left\{\frac{\pi_{t}^{2}}{2}+\lambda_{x} \frac{x_{t}^{2}}{2}+\lambda_{i} \frac{i_{t}^{2}}{2}+\phi_{x, t}\left[x_{t}-x_{t+1}+\sigma\left(i_{t}-\pi_{t+1}\right)\right]+\phi_{\pi, t}\left[\pi_{t}-\beta_{t} \pi_{t+1}-\kappa x_{t}\right]\right\}$

The interest rate (policy instrument) is a linear decreasing function of the Lagrange multiplier of the output gap $\phi_{x, t}$ :

$$
\frac{\partial \mathcal{L}}{\partial i_{t}}=0 \Rightarrow \lambda_{i} i_{t}+\sigma \phi_{x, t}=0 \Rightarrow \phi_{x, t}=-\frac{\lambda_{i}}{\sigma} i_{t} \text { or } i_{t}=-\frac{\sigma}{\lambda_{i}} \phi_{x, t} \text { for } t=0,1,2, \ldots
$$

Because inflation and the output gap are forward-looking variables, they are optimally chosen at the initial date $t=-1$ and at the final date according to transversality conditions. The marginal values of the loss function with respect to output gap $\phi_{x, t=-1}$ and to inflation $\phi_{\pi, t=-1}$ are equal to zero at the initial date:

$$
\phi_{\pi, t=-1}=\phi_{x, t=-1}=0 \text { and } \lim _{t \rightarrow+\infty} \phi_{\pi, t}=\lim _{t \rightarrow+\infty} \phi_{x, t}=0 .
$$

Because the marginal value of the loss function with respect to the output gap at the initial date $t=-1$ is zero $\left(\phi_{x, t=-1}=0\right)$, the initial interest rate is set to zero: $i_{-1}=0$. 
The Hamiltonian system includes the Euler consumption equation (1), the newKeynesian Phillips curve (3) and the two first order conditions with respect to output gap and to inflation:

$$
\begin{aligned}
& \frac{\partial \mathcal{L}}{\partial x_{t}}=0 \Rightarrow \lambda_{x} x_{t}+\phi_{x, t}-\beta^{-1} \phi_{x, t-1}-\kappa \phi_{\pi, t}=0 \text { for } t=1,2, \ldots \\
& \frac{\partial \mathcal{L}}{\partial \pi_{t}}=0 \Rightarrow \pi_{t}-\beta^{-1} \sigma \phi_{x, t-1}+\phi_{\pi, t}-\phi_{\pi, t-1}=0 \text { for } t=1,2, \ldots
\end{aligned}
$$

One considers two observationally equivalent representations of the Hamiltonian system using linear substitution between variables across equations. The most common representation substitutes the policy instruments by the Lagrange multipliers: the interest rate $i_{t}$ in the Euler consumption equation (1) is substituted by $\phi_{x, t}$ (Hansen and Sargent (2008), chapter 3). Then, the Hamiltonian system involves four variables: output gap and inflation $\left(x_{t}, \pi_{t}\right)$ and their Lagrange multipliers $\left(\phi_{x, t}, \phi_{\pi, t}\right)$ and four equations, with a symplectic Hamiltonian matrix: its transpose is similar to its inverse. This matrix has an equal number of stable eigenvalues and of unstable eigenvalues, which is equal to two in this case. The number of stable eigenvalues is equal to the number of predetermined variables $\left(\phi_{x, t}, \phi_{\pi, t}\right)$ which corresponds to predetermined values of the policy instrument and of its first lag $\left(i_{t}, i_{t-1}\right)$. The number of unstable eigenvalues is equal to the number of forward-looking variables $\left(x_{t}, \pi_{t}\right)$. Blanchard and Kahn (1980) determinacy condition is satisfied: the number of stable eigenvalue is equal to the number of predetermined variables. Blanchard and Kahn (1980) acknowledged that the determinacy condition is always satisfied in the general LQR case and in optimal growth models. In addition, the non-controllable part of the economy has two stable eigenvalues $\left(\rho_{z}, \rho_{u}\right)$ which are the auto-correlation parameters of two predetermined forcing variables $\left(z_{t}, u_{t}\right)$, so that Blanchard and Kahn (1980) determinacy condition is satisfied.

An alternative representation of the Hamiltonian system is to substitute the Lagrange multipliers by the policy instrument. If the number of instruments is smaller than the number of policy targets or the number of their Lagrange multipliers, one includes lags of the policy instrument. Linear substitutions of the Lagrange multipliers $\left(\phi_{x, t}, \phi_{\pi, t}\right)$ by the policy instrument and its lag $\left(i_{t}, i_{t-1}\right)$ leads to an equivalent Hamiltonian linear dynamic system with variables $\left(x_{t}, \pi_{t}, i_{t}, i_{t-1}\right)$.

In the case of an optimal commitment for Ramsey policy that has been in force since at least period $t-2\left(\phi_{x, t-3}=\phi_{\pi, t-3}=0\right.$ and $\left.i_{t-3}=0\right)$, GW reasoning is that "one can infer the value of the Lagrange multiplier $\phi_{x, t-1}$ from the value of $i_{t-1}$ using Equation (7), and similarly, the value of $\phi_{x, t-2}$ from the value of $i_{t-2}$ :

$$
\frac{\partial \mathcal{L}}{\partial i_{t}}=0 \Rightarrow \phi_{x, t}=-\frac{\lambda_{i}}{\sigma} i_{t} \text { for } t=-2,-1,0,1, \ldots
$$

Then, substituting theses values into Equation (9) for period $t-1$, one can also infer the value of $\phi_{\pi, t-1}$ from the value of $x_{t-1}$. One can, of course, similarly solve for the period $t$ Lagrange multipliers as functions of $x_{t}, i_{t}$ and $i_{t-1}$ :

$$
\frac{\partial \mathcal{L}}{\partial x_{t}}=0 \Rightarrow \phi_{\pi, t}=\frac{1}{\kappa} \lambda_{x} x_{t}-\frac{1}{\kappa} \frac{\lambda_{i}}{\sigma} i_{t}+\frac{\beta^{-1}}{\kappa} \frac{\lambda_{i}}{\sigma} i_{t-1} \text { for } t=-1,0,1, \ldots
$$

Using these expressions to substitute out the Lagrange multipliers in equation (10), one obtains a linear relation among the endogenous variables $\pi_{t}, x_{t}, x_{t-1}, i_{t-1}, i_{t-1}$ and 
$i_{t-2}$ that must hold for any period $t \geq 0$, for an optimal commitment for Ramsey policy that has been in force since at least period -2 :

$$
i_{t}=\left(1+\frac{\kappa \sigma}{\beta}\right) i_{t-1}+\frac{1}{\beta} \Delta i_{t-1}+\frac{\kappa \sigma}{\lambda_{i}} \pi_{t}+\frac{4 \sigma \lambda_{x}}{\lambda_{i}} \frac{\Delta x_{t}}{4} \text { for } t=0,1,2, \ldots
$$

GW label "super-inertial" this representation (13) of the first order condition of inflation (10): the implied dynamics of the funds rate are explosive, because the sum of parameters for lagged funds rate exceeds one: $1+\frac{\kappa \sigma}{\beta}>1$.

GW proposition 1 states that commitment to this super-inertial policy rule (13) is a necessary condition which implies determinacy. They prove that their representation of the Hamiltonian system including the super-inertial policy rule (13) has a number of stable eigenvalues equal to the number of predetermined variables (GW appendix one). As mentioned above, this is usually demonstrated with the previous representation of the Hamiltonian system with variables $\left(x_{t}, \pi_{t}\right)$ and their Lagrange multipliers $\left(\phi_{x, t}, \phi_{\pi, t}\right)$ in the general LQR case.

This super-inertial rule (13) corresponds to the unique bounded solution and to an infinity of out-of-equilibrium unbounded paths with dynamics for the funds rate which are explosive. At this stage, GW proved that their super-inertial policy (the first order condition on inflation) is a necessary condition to this unique bounded solution but not a sufficient condition ruling out unbounded paths.

GW, p.1430, just below proposition 1, state without proof that their super-inertial policy is ruling out unbounded paths: "The equilibrium determined by commitment to this rule (13) from date $t=0$ onwards corresponds to the unique bounded solution to equations $(1,2,3,4,7,9,10)$ when the initial condition $\left((8): \phi_{x,-1}=\phi_{\pi,-1}=0\right.$ and $\left.i_{t-1}=0\right)$ is replaced by the values of $\phi_{x,-1}$ and $\phi_{\pi,-1}$ that would be inferred from the historical values of $x_{-1}, i_{-1}$, and $i_{-2}$ under the reasoning described above."

The change of initial conditions corresponds to the case of an optimal commitment for Ramsey policy that has been in force since at least period $t-2\left(\phi_{x, t-3}=\phi_{\pi, t-3}=0\right.$ and $\left.i_{t-3}=0\right)$ instead of instead of period $t=0\left(\phi_{x,-1}=\phi_{\pi,-1}=0\right.$ and $\left.i_{t-1}=0\right)$. The super-inertial rule (13) is then valid after two periods starting at $t \geq 0$. The "reasoning described above" is the substitution of the Lagrange multipliers by the interest rate and its two lags in the first order equation of inflation. These practical informations do not rule out the unbounded paths predicted by the super-inertial rule (13).

Once the determinacy condition is fulfilled (GW proposition 1), Blanchard and Kahn (1980) and Vaughan (1970) demonstrated that the unique bounded solution and the optimal policy rule are obtained seeking the stable subspace of the Hamiltonian system using the method of undetermined coefficients or, equivalently, solving a matrix Riccati equation which amounts to seek the eigenvectors of stable roots of a Jordan decomposition of the Hamiltonian matrix.

\section{Optimal inertial policy rules}

\subsection{Clarida Gali and Gertler (1999) case.}

Proposition 1 Clarida, Gali and Gertler (1999). The equilibrium determined by commitment from date $t=0$ onwards to an inertial output gap rule, where the auto-correlation of the output gap is below one, corresponds to the unique bounded solution of Ramsey 
optimal policy for the new-Keynesian Phillips curve (3) and the cost-push shock (4)

Proof. In Clarida Gali and Gertler (1999) and Gali (2015, chapter 5) optimization of Ramsey optimal policy, the intertemporal substitution equation of consumption (1) and its auto-correlated shock equation (2) are not taken into account, so that there is no interest rate smoothing: $i_{t}=0, \lambda_{i}=0, \phi_{x}=0$ and $z_{t}=0$. The output gap $x_{t}$ plays the role of the policy instrument in a "targeting rule": $\lambda_{x}>0$. Inflation is the only policy target. The Hamiltonian system includes the new-Keynesian Phillips curve and the noncontrollable dynamics of the cost-push shock. The marginal equation with respect to the single policy instrument (output gap) implies that it is a linear increasing function of the Lagrange multiplier of inflation:

$$
\frac{\partial \mathcal{L}}{\partial x_{t}}=0 \Rightarrow \lambda_{x} x_{t}-\kappa \phi_{\pi, t}=0 \text { for } t=0,1,2, \ldots \Rightarrow x_{t}=\frac{\kappa}{\lambda_{x}} \phi_{\pi, t} \text { or } \phi_{\pi, t}=\frac{\lambda_{x}}{\kappa} x_{t}
$$

The initial natural boundary initial sets to zero the initial value of the policy instrument at the initial date -1 :

$$
\phi_{\pi,-1}=0 \Rightarrow x_{-1}=-\frac{\kappa}{\lambda_{x}} \phi_{\pi,-1}=0
$$

The first order condition on inflation is:

$$
\frac{\partial \mathcal{L}}{\partial \pi_{t}}=0 \Rightarrow \pi_{t}+\phi_{\pi, t}-\phi_{\pi, t-1}=0 \text { for } t=0,1,2, \ldots
$$

One substitutes the Lagrange multiplier of inflation $\phi_{\pi, t}$ by the policy instrument $x_{t}$ :

$$
\frac{\partial \mathcal{L}}{\partial \pi_{t}}=0 \Rightarrow x_{t}=x_{t-1}-\frac{\kappa}{\lambda_{x}} \pi_{t} \text { for } t=0,1,2,3 \ldots
$$

This equation corresponds to GW super-inertial policy rule. The policy instrument (the output gap) has unit root non-stationary dynamics. When time goes to infinity, this non-stationary policy rule allows an infinity of unbounded out-of-equilibrium paths with an infinite variance of the policy instrument and a unique bounded stationary solution. Clarida, Gali and Gertler (1999) and Gali (2015) seek the unique stable eigenvalue $\delta$ of the Hamiltonian system:

$$
0<\delta=\frac{1}{2}\left(1+\frac{1}{\beta}+\frac{\kappa^{2}}{\beta \lambda_{x}}\right)-\sqrt{\frac{1}{4}\left(1+\frac{1}{\beta}+\frac{\kappa^{2}}{\beta \lambda_{x}}\right)^{2}-\frac{1}{\beta}}<1
$$

The Hamiltonian system satisfies Blanchard and Kahn (1980) determinacy condition (as GW proposition one): two stable eigenvalues $\delta$ and $\rho_{u}$ for two predetermined variables $\phi_{\pi, t}$ and $u_{t}$. By contrast to GW, Clarida, Gali and Gertler (1999) and Gali (2015) go one step further. They find the optimal stationary inertial policy rule using the method of undetermined coefficients:

$$
x_{t}=\delta x_{t-1}-\frac{\delta}{1-\delta \beta \rho_{u}} \frac{\kappa}{\lambda_{x}} u_{t} \text { for } t=1,2, \ldots \text { and } x_{0}=-\frac{\delta}{1-\delta \beta \rho_{u}} \frac{\kappa}{\lambda_{x}} u_{0}
$$

The representation of the inertial optimal policy rule for the output gap has an autoregressive component $\delta$ which is strictly lower than one. It also depends on the auto-correlation of the cost push shock $\rho_{u}$ and on the shock $u_{t}$. One can find another representation of this 
inertial policy rule after substitution of the cost-push shock $u_{t}$ by inflation $\pi_{t}$ (Chatelain and Ralf 2017a)).

\subsection{Inertial optimal policy rule}

Proposition 2 The equilibrium determined by commitment from date $t=0$ onwards to an inertial interest rate rule, where the sum of the parameters of lags of interest rate is below one and depends on the auto-correlation of shocks, corresponds to the unique bounded solution of Ramsey optimal policy for the new-Keynesian model (equations 1,2,3,4).

Proof. Hansen and Sargent (2008) section 3.5 and Anderson et al. (1996) solution of this discounted LQR augmented with forcing variables $\left(z_{t}, u_{t}\right)$ solves a discrete algebraic Riccati equation for rule parameters $\left(F_{x}, F_{\pi}\right)$ which depend on parameters $(\beta, \kappa, \sigma)$ and preferences $\left(\lambda_{x}, \lambda_{i}\right)$ but do not depend on the auto-correlation of shocks and solves a Sylvester equation for rule parameters $\left(F_{z}, F_{u}\right)$ which depends on the auto-correlation of shocks $\left(\rho_{z}, \rho_{u}\right)$ :

$$
i_{t}=F_{x} x_{t}+F_{\pi} \pi_{t}+F_{z} z_{t}+F_{u} u_{t}
$$

There is no closed form solution for these parameters, only numerical solutions. Doing linear substitutions within the system of equations including the policy rule (17) and the private sector equations $(1,2,3,4)$ leads to an equivalent system with observational equivalence. Applying the operator $p(L)=\left(1-\rho_{u} L\right)\left(1-\rho_{z} L\right)$, where $L$ is the lag operator, on Hansen and Sargent (2008) optimal rule (17) obtains an observationally equivalent representation (18) of the optimal policy rule (17), where the two auto-regressive forcing variables $z_{t}$ and $u_{t}$ are substituted by the two first lags of the policy instrument: $i_{t-1}$ and $i_{t-2}$ :

$$
p(L) i_{t}=F_{x} p(L) x_{t}+F_{\pi} p(L) \pi_{t}+F_{z}\left(1-\rho_{u} L\right) \varepsilon_{z, t}+F_{u}\left(1-\rho_{z} L\right) \varepsilon_{u, t}
$$

The sum of the parameters of the two lags of the dependent variables is the sum $S$ less the product $P$ of the two auto-correlation coefficients of shocks:

$$
i_{t}=\left(\rho_{z}+\rho_{u}\right) i_{t-1}-\left(\rho_{z} \rho_{u}\right) i_{t-2}+\ldots
$$

Because the two auto-correlation coefficients are between zero and one and are the roots of the polynomial $p, p(1)>0$. Hence, the sum of the two parameters of the lags of the interest rate is strictly below one:

$$
p(1)=1-S+P>0 \Rightarrow S-P<1
$$

Hence, the observationally equivalent representation (18) of the optimal policy rule (17) is inertial. It depends on the auto-correlation of shocks.

Simon (1956) proved that the optimal rule parameters of the LQR are independent of the disturbances of the shocks $\left(\varepsilon_{z, t}, \varepsilon_{u, t}\right)$, in particular of their variance, and of the initial conditions of private sectors variables $\left(x_{0}, \pi_{0}, z_{0}, u_{0}\right)$. Using the optimal initial condition $\left(x_{0}^{*}, \pi_{0}^{*}\right)$ or ad hoc initial jumps for forward-looking variables $\left(x_{0}, \pi_{0}\right)$ such as timeless perspective or predetermined values for $\left(x_{0}, \pi_{0}\right)$ do not change the optimal policy rule parameters. One finds the optimal initial conditions $\left(x_{0}^{*}, \pi_{0}^{*}\right)$ of Ramsey optimal policy using Ljungqvist and Sargent (2012) chapter 19 and Chatelain and Ralf (2017b) algorithm. 


\section{Conclusion}

Ramsey optimal policy for the new-Keynesian model implies a stationary optimal policy rule which is not super-inertial and which depends on the auto-correlation of shocks.

\section{References}

[1] Anderson E.W., Hansen L.P., McGrattan E.R. and Sargent T.J. (1996). Mechanics of Forming and Estimating Dynamic Linear Economies. in Amman H.M., Kendrick D.A. and Rust J. (editors) Handbook of Computational Economics, Elsevier, Amsterdam, 171-252.

[2] Adolfson, M., Laséen, S., Lindé, J., \& Svensson, L. E. (2014). Monetary policy tradeoffs in an estimated open-economy DSGE model. Journal of Economic Dynamics and Control, 42, 33-49.

[3] Blanchard, O. J., \& Kahn, C. M. (1980). The solution of linear difference models under rational expectations. Econometrica: Journal of the Econometric Society, 1305-1311.

[4] Clarida, R., Gali, J., \& Gertler, M. (1999). The science of monetary policy: a new Keynesian perspective. Journal of economic literature, 37(4), 1661-1707.

[5] Chatelain J.B. and Ralf K. (2014). Stability and Identification with Optimal Macroprudential Policy Rules. MPRA working paper.

[6] Chatelain, J. B., and Ralf, K. (2014). Peut-on identifier les politiques économiques stabilisant une économie instable? Revue française d'économie, 29, pp. 143-178.

[7] Chatelain J.B. and Ralf K. (2014). A Finite Set of Equilibria for the Indeterminacy of Linear Rational Expectations Models. MPRA paper.

[8] Chatelain J.B. and Ralf K. (2016). Countercyclical versus Procyclical Taylor Principles. SSRN working paper.

[9] Chatelain, J. B., \& Ralf, K. (2017a). Can we Identify the Fed's preferences? PSE and SSRN working paper.

[10] Chatelain, J. B., \& Ralf, K. (2017b). A Simple Algorithm for Solving Ramsey Optimal Policy with Exogenous Forcing Variables. PSE and SSRN working paper.

[11] Chatelain, J. B., \& Ralf, K. (2018a). Imperfect Credibility versus No Credibility of Optimal Monetary Policy. PSE and SSRN working paper.

[12] Chatelain, J. B., \& Ralf, K. (2018b). Hopf Bifurcation from New-Keynesian Taylor Rule to Ramsey Optimal Policy. PSE and SSRN working paper.

[13] Chatelain, J. B., \& Ralf, K. (2018c). The Indeterminacy of Determinacy with Fiscal, Macro-prudential or Taylor Rules. PSE and SSRN working paper.

[14] Chung, H., Herbst, E., \& Kiley, M. T. (2015). Effective Monetary Policy Strategies in New Keynesian Models: A Reexamination. NBER Macroeconomics Annual, 29(1), 289-344. 
[15] Gali J. (2015). Monetary Policy, Inflation and the Business Cycle. 2nd edition, Princeton University Press, Princeton.

[16] Giannoni, M. P. (2014). Optimal interest-rate rules and inflation stabilization versus price-level stabilization. Journal of Economic Dynamics and Control, 41, 110-129.

[17] Giannoni, M. P. \& Woodford M. (2003). How forward-looking is optimal monetary policy? Journal of Money, Credit, and Banking, 35(6), 1425-1469.

[18] Hansen L.P. and Sargent T. (2008). Robustness, Princeton University Press, Princeton.

[19] Ljungqvist L. and Sargent T.J. (2012). Recursive Macroeconomic Theory. 3rd edition. The MIT Press. Cambridge, Massaschussets.

[20] Simon, H. A. (1956). Dynamic programming under uncertainty with a quadratic criterion function. Econometrica, Journal of the Econometric Society, 74-81. 